\title{
A DIAGNOSTIC SPECTRAL INDICATOR OF THE EXPOSURE AGE OF AN ASTEROIDAL SURFACE
}

\author{
Joseph A. Nuth III \\ Astrochemistry Branch, Code 691 \\ NASA/Goddard Space Flight Center \\ Greenbelt, MD 20771
}

The energy of the $\mathrm{SiH}$ vibrational fundamental has been shown be extremely sensitive to the oxidation state of the silicon to which the hydrogen is bound, ranging from 4.4 microns in highly oxidized silicate grains to 4.74 microns in silicon carbide (Moore, Tanabé and Nuth, Ap. J. (Lett.) 373, L31-L34, 1991). Yin, Ghose and Adler (Appl. Spectrosc. 26, 355-7, 1972) have shown that the process of ion-sputtering a metal oxide results in chemical reduction, due to the high sputtering yield and volatility of oxygen relative to the metal. These authors have shown that solar wind ion-sputtering of lunar soils could provide an explanation for the observed solar wind darkening of the lunar surface. We hypothesize that a similar ion-reduction process could occur on asteroidal surfaces exposed to the solar wind. A second consequence of solar wind exposure would be the implantation of hydrogen ions into the asteroidal surface. Stein (J. Elec. Mat. 4, 159-174, 1975) has shown that hydrogen-ions implanted into silicon exhibit infrared absorptions between 4.5 and 5.5 microns: similar features should result from the ion-implantation of hydrogen ions into partially reduced silicate minerals and glasses. The position of such features would be indicative of the local chemical environment of the silicon atom to which the hydrogen was bound.

It may be possible to observe the $\mathrm{SiH}$ fundamental on the lunar surface, however, laboratory experiments have shown that exposure of grains containing-SiH groups to vacuum for several months at $370 \mathrm{~K}$ results in the elimination of hydrogen from the grains. Therefore, for lunar surfaces where the temperature might exceed $300 \mathrm{~K}$ for a considerable length of time each lunar day, it is possible that the $\mathrm{SiH}$ signature could be baked out if the rate of solar wind ionimplantation does not exceed the rate at which hydrogen is thermally driven from the grains. Nevertheless, as long as some $\mathrm{SiH}$ still exists in the lunar regolith, the wavelength of the $\mathrm{SiH}$ fundamental will be indicative of the oxidation state of the silicon in the surface of the grains.

Asteroidal regoliths will be considerably cooler than the lunar surface due to their greater distance from the sun and this should ensure that such regoliths will retain solar wind implanted hydrogen ions. Of course, increased distance of the asteroids from the sun decreases both the solar wind hydrogen ion implantation rate and the rate of ion-sputtering reduction of the asteroidal regolith. This will mean that surfaces require quite long exposure to the solar wind to become reduced. Exact correlation of the solar wind exposure ion-sputtering reduction age with the energy of the $\mathrm{SiH}$ fundamental requires careful laboratory studies, which have yet to be performed, of the reduction efficiency of solar wind ions for various meteorite types. These efficiencies would then be integrated over the orbit of the asteroid and corrected for variations in the solar wind flux through time in order to calculate absolute ages. 
Despite the difficulty in obtaining absolute ages for asteroidal surfaces, it should be possible to obtain relative ages for asteroids of similar type in similar orbits quite easily if the peak frequency of the $\mathrm{SiH}$ fundamental can be determined. The surfaces of such asteroids should have received similar doses of solar wind irradiation over time and this radiation should have produced the same degree of chemical reduction in each. However, if due to collision a fresh surface on a chondritic-type asteroid were exposed, then this surface would be fully oxidized and one would expect the SiH fundamental to occur near $2300 \mathrm{~cm}^{-1}$ once a suitable quantity of hydrogen had been implanted into the surface to be observable. As the surface ages and becomes more reduced the energy of the $\mathrm{SiH}$ fundamental would gradually decrease towards $2100 \mathrm{~cm}^{-1}$. The youngest surface is therefore the one with the highest energy $\mathrm{SiH}$ fundamental. For relatively slow rotating asteroids it may even be possible to determine the relative ages of different surfaces provided that sufficiently high signal-to-noise spectra can be obtained on a timescale which is short in comparison to the rotation period. Such data may not obtainable in this difficult spectral region until space-based infrared observatories become available. 